\title{
Norms over intuitionistic fuzzy congruence relations on rings
}

\author{
Rasul Rasuli \\ Department of Mathematics, Payame Noor University (PNU) \\ Tehran, Iran \\ e-mail: rasulirasuleyahoo.com
}

Received: 20 June 2021

Accepted: 27 August 2021

\begin{abstract}
In this paper, by using norms, we define the concept of intuitionistic fuzzy equivalence relations and intuitionistic fuzzy congruence relations on ring $\mathrm{R}$ and we investigate some assertions. Also we define intuitionistic fuzzy ideals of ring $\mathrm{R}$ under norms and compare this with fuzzy equivalence relation and fuzzy congruence relation on ring $\mathrm{R}$ such that we define new introduced ring.
\end{abstract}

Keywords: Rings, Ideals, Congruence relations, Fuzzy set theory, Intuitionistic mathematics, Norms, Bifuzzy equivalence relation, Bifuzzy congruence relation, Bifuzzy ideals.

2020 Mathematics Subject Classification: 05C25, 06B10, 03E72, 47A30.

\section{Introduction}

In algebra, ring theory is the study of rings algebraic structures in which addition and multiplication are defined and have similar properties to those operations defined for the integers. Ring theory studies the structure of rings, their representations, or, in different language, modules, special classes of rings (group rings, division rings, universal enveloping algebras), as well as an array of properties that proved to be of interest both within the theory itself and for its applications, such as homological properties and polynomial identities. In ring theory, a branch of abstract algebra, an ideal is a special subset of a ring. Ideals generalize certain subsets of the integers, such as the even numbers or the multiples of 3 . Addition and subtraction of even numbers preserves evenness, and multiplying an even number by any other integer results in another even number; 
these closure and absorption properties are the defining properties of an ideal. An ideal can be used to construct a quotient ring similarly to the way that, in group theory, a normal subgroup can be used to construct a quotient group. In mathematics, fuzzy sets (aka uncertain sets) are somewhat like sets whose elements have degrees of membership. Fuzzy sets were introduced independently by Zadeh and Dieter Klaua [5, 48] as an extension of the classical notion of set. In classical set theory, the membership of elements in a set is assessed in binary terms according to a bivalent condition an element either belongs or does not belong to the set. By contrast, fuzzy set theory permits the gradual assessment of the membership of elements in a set; this is described with the aid of a membership function valued in the real unit interval $[0,1]$. Fuzzy sets generalize classical sets, since the indicator functions (also known as characteristic functions) of classical sets are special cases of the membership functions of fuzzy sets, if the latter only take values 0 or 1 [4]. In fuzzy set theory, classical bivalent sets are usually called crisp sets. The fuzzy set theory can be used in a wide range of domains in which information is incomplete or imprecise, such as bioinformatics [6]. Intuitionistic set theory is a set theory, generally proffered as a foundation of mathematics, intended to capture intuitionistic mathematics. As the terminology is usually used (for example in the name of IZF, intuitionistic Zermelo-Frankel set theory), 'intuitionistic' means that excluded middle fails but power sets are included (making it impredicative). In contrast, 'constructive' set theory (such as CZF, constructive Zermelo-Frankel set theory) has function sets but not power sets (making it weakly predicative). The former is technically convenient, but the latter is better motivated. That said, Brouwer's mathematics was even more predicative, making both of these set theories stronger than he would accept. Triangular norms and conorms are operations which generalize the logical conjunction and logical disjunction to fuzzy logic. They are a natural interpretation of the conjunction and disjunction in the semantics of mathematical fuzzy logics and they are used to combine criteria in multi-criteria decision making. The author by using norms, investigated some properties of fuzzy algebraic structures [8-47]. In this study, by using norms, we introduce intuitionistic fuzzy equivalence relations, intuitionistic fuzzy congruence relations and intuitionistic fuzzy ideals on ring $R$ and obtain some results about them. Also we investigate some of their properties and structured characteristics of them.

\section{Preliminaries}

This section contains some basic definitions and preliminary results which will be needed in the sequal. For more details we refer to $[1,2,3,7]$.

Definition 2.1. A ring $\langle R,+,$.$\rangle consists of a nonempty set R$ and two binary operations + and . that satisfy the axioms:

(1) $\langle R,+,$.$\rangle is an Abelian group;$

(2) $(a b) c=a(b c)$ (associative multiplication) for all $a, b, c \in R$;

(3) $a(b+c)=a b+a c,(b+c) a=b a+c a$ (distributive laws) for all $a, b, c \in R$

Moreover, the ring $R$ is a commutative ring if $a b=b a$ and ring with identity if $R$ contains an element $1_{R}$ such that $1_{R} a=a 1_{R}=$ a for all $a \in R$. 
Example 2.2. (1) The ring $\mathbb{Z}$ of integers is a commutative ring with identity. So are $\mathbb{Q}, \mathbb{R}, \mathbb{C}, \mathbb{Z}_{n}$, $\mathbb{R}[x]$, etc.

(2) $5 \mathbb{Z}$ is a commutative ring with no identity.

(3) The ring $\mathbb{Z}^{3 \times 3}$ of $3 \times 3$ matrices with integer coefficients is a noncommutative ring with identity.

(4) $(5 \mathbb{Z})^{3 \times 3}$ is a noncommutative ring with no identity.

Definition 2.3. Let $X$ be a non-empty sets. A fuzzy subset $\mu$ of $X$ is a function $\mu: X \rightarrow[0,1]$. Denote by $[0,1]^{X}$, the set of all fuzzy subsets of $X$.

Definition 2.4. For sets $X, Y$ and $Z, f=\left(f_{1}, f_{2}\right): X \rightarrow Y \times Z$ is called a complex mapping if $f_{1}: X \rightarrow Y$ and $f_{2}: X \rightarrow Z$ are mappings.

Definition 2.5. Let $X$ be a nonempty set. A complex mapping $A=\left(\mu_{A}, \nu_{A}\right): X \rightarrow[0,1] \times[0,1]$ is called an intuitionistic fuzzy set (in short, IFS) in $X$ such that $\mu_{A}, \nu_{A} \in[0,1]^{X}$ and for all $x \in X$ we have $\left(\mu_{A}(x)+\nu_{A}(x)\right) \in[0,1]$. In particular, $\emptyset_{X}$ and $U_{X}$ denote the intuitionistic fuzzy empty set and intuitionistic fuzzy whole set in $X$ defined by $\emptyset_{X}(x)=(0,1)$ and $U_{X}(x)=(1,0)$, respectively. We will denote the set of all IFSs in X by IFS $(X)$.

Definition 2.6. Let $X$ be a nonempty set and let $A=\left(\mu_{A}, \nu_{A}\right)$ and $B=\left(\mu_{B}, \nu_{B}\right)$ be IFSs in X. Then

(1) Inclusion: $A \subseteq B$ iff $\mu_{A} \leq \mu_{B}$ and $\nu_{A} \geq \nu_{B}$.

(2) Equality: $A=B$ iff $A \subseteq B$ and $B \subseteq A$.

Definition 2.7. A t-norm $T$ is a function $T:[0,1] \times[0,1] \rightarrow[0,1]$ having the following four properties:

(T1) $T(x, 1)=x$ (neutral element)

(T2) $T(x, y) \leq T(x, z)$ if $y \leq z$ (monotonicity)

(T3) $T(x, y)=T(y, x)$ (commutativity)

(T4) $T(x, T(y, z))=T(T(x, y), z)$ (associativity),

for all $x, y, z \in[0,1]$.

Corollary 2.8. Let $T$ be a $T$-norm. Then for all $x \in[0,1]$

(1) $T(x, 0)=0$.

(2) $T(0,0)=0$.

Example 2.9. (1) Standard intersection $T$-norm $T_{m}(x, y)=\min \{x, y\}$.

(2) Bounded sum T-norm $T_{b}(x, y)=\max \{0, x+y-1\}$.

(3) Algebraic product $T$-norm $T_{p}(x, y)=x y$.

(4) Drastic T-norm:

$$
T_{D}(x, y)= \begin{cases}y, & \text { if } x=1 \\ x, & \text { if } y=1 \\ 0, & \text { otherwise. }\end{cases}
$$

(5) Nilpotent minimum T-norm:

$$
T_{n M}(x, y)=\left\{\begin{aligned}
\min \{x, y\}, & \text { if } x+y>1 \\
0, & \text { otherwise }
\end{aligned}\right.
$$


(6) Hamacher product T-norm:

$$
T_{H_{0}}(x, y)=\left\{\begin{aligned}
0, & \text { if } x=y=0 \\
\frac{x y}{x+y-x y}, & \text { otherwise. }
\end{aligned}\right.
$$

The drastic $t$-norm is the pointwise smallest $t$-norm and the minimum is the pointwise largest $t$-norm: $T_{D}(x, y) \leq T(x, y) \leq T_{\min }(x, y)$ for all $x, y \in[0,1]$.

Lemma 2.1. Let $T$ be a t-norm. Then

$$
T(T(x, y), T(w, z))=T(T(x, w), T(y, z)),
$$

for all $x, y, w, z \in[0,1]$.

Definition 2.10. A t-conorm $C$ is a function $C:[0,1] \times[0,1] \rightarrow[0,1]$ having the following four properties:

(C1) $C(x, 0)=x$

(C2) $C(x, y) \leq C(x, z)$ if $y \leq z$

(C3) $C(x, y)=C(y, x)$

(C4) $C(x, C(y, z))=C(C(x, y), z)$,

for all $x, y, z \in[0,1]$.

Corollary 2.11. Let $C$ be a $C$-conorm. Then for all $x \in[0,1]$ :

(1) $C(x, 1)=1$.

(2) $C(0,0)=0$.

Example 2.12. (1) Standard union t-conorm $C_{m}(x, y)=\max \{x, y\}$.

(2) Bounded sum t-conorm $C_{b}(x, y)=\min \{1, x+y\}$.

(3) Algebraic sum t-conorm $C_{p}(x, y)=x+y-x y$.

(4) Drastic T-conorm

$$
C_{D}(x, y)= \begin{cases}y, & \text { if } x=0 \\ x, & \text { if } y=0 \\ 1, & \text { otherwise }\end{cases}
$$

dual to the drastic T-norm.

(5) Nilpotent maximum T-conorm, dual to the nilpotent minimum T-norm:

$$
C_{n M}(x, y)=\left\{\begin{aligned}
\max \{x, y\}, & \text { if } x+y<1 . \\
1, & \text { otherwise. }
\end{aligned}\right.
$$

(6) Einstein sum (compare the velocity-addition formula under special relativity) $C_{H_{2}}(x, y)=$ $\frac{x+y}{1+x y}$ is a dual to one of the Hamacher t-norms. Note that all t-conorms are bounded by the maximum and the drastic t-conorm: $C_{\max }(x, y) \leq C(x, y) \leq C_{D}(x, y)$ for any $t$-conorm $C$ and all $x, y \in[0,1]$.

Recall that $t$-norm $T(t$-conorm $C)$ is idempotent if for all $x \in[0,1], T(x, x)=x(C(x, x)=x)$.

Lemma 2.2. Let $C$ be a t-conorm. Then

$$
C(C(x, y), C(w, z))=C(C(x, w), C(y, z)),
$$

for all $x, y, w, z \in[0,1]$. 


\section{Norms over intuitionistic fuzzy congruence relations on rings}

Throughout this paper, $R$ stands for the ring $\langle R,+,$.$\rangle with an identity element 1_{R}$ and zero element $0_{R}$ such that it is commutative.

Definition 3.1. An intuitionistic fuzzy relation $A=\left(\mu_{A}, \nu_{A}\right) \in I F S(R \times R)$ is called an intuitionistic fuzzy equivalence relation on $R$ under norms ( $T$ and $C$ ), if it satisfies:

(1) $\mu_{A}(x, x)=1$,

(2) $\mu_{A}(x, y)=\mu_{A}(y, x)$,

(3) $\mu_{A}(x, z) \geq T\left(\mu_{A}(x, y), \mu_{A}(y, z)\right)$,

(4) $\nu_{A}(x, x)=0$,

(5) $\nu_{A}(x, y)=\nu_{A}(y, x)$,

(6) $\nu_{A}(x, z) \leq C\left(\nu_{A}(x, y), \nu_{A}(y, z)\right)$,

for all $x, y, z \in R$.

Definition 3.2. An intuitionistic fuzzy relation $A=\left(\mu_{A}, \nu_{A}\right) \in I F S(R \times R)$ is called an intuitionistic fuzzy congruence relation on $R$ under norms ( $T$ and $C$ ), if it satisfies:

(1) $\mu_{A}(x, x)=1$,

(2) $\mu_{A}(x, y)=\mu_{A}(y, x)$,

(3) $\mu_{A}(x, z) \geq T\left(\mu_{A}(x, y), \mu_{A}(y, z)\right)$,

(4) $\mu_{A}(x+z, y+t) \geq T\left(\mu_{A}(x, y), \mu_{A}(z, t)\right)$,

(5) $\mu_{A}(x z, y t) \geq T\left(\mu_{A}(x, y), \mu_{A}(z, t)\right)$,

(6) $\nu_{A}(x, x)=0$,

(7) $\nu_{A}(x, y)=\nu_{A}(y, x)$,

(8) $\nu_{A}(x, z) \leq C\left(\nu_{A}(x, y), \nu_{A}(y, z)\right)$,

(9) $\nu_{A}(x+z, y+t) \leq C\left(\nu_{A}(x, y), \nu_{A}(z, t)\right)$,

(10) $\nu_{A}(x z, y t) \leq C\left(\nu_{A}(x, y), \nu_{A}(z, t)\right)$,

for all $x, y, z, t \in R$.

We denote the set of all intuitionistic fuzzy congruence relations on $R$ under norms $(T$ and $C)$ by $\operatorname{IFCN}(R \times R)$.

Example 3.3. Let $R=(\mathbb{Z},+,$.$) be a ring of integer numbers. Define a bifuzzy relation$ $A=\left(\mu_{A}, \nu_{A}\right) \in I F S(R \times R) b y$

$$
\mu_{A}(x, y)=\left\{\begin{aligned}
1, & \text { if } x=y \\
0.65, & \text { otherwise }
\end{aligned}\right.
$$

and

$$
\nu_{A}(x, y)=\left\{\begin{aligned}
0, & \text { if } x=y . \\
0.85, & \text { otherwise. }
\end{aligned}\right.
$$

If $T(x, y)=T_{m}(x, y)=\min \{x, y\}$ and $C_{p}(x, y)=x+y-x y$ for all $x, y \in[0,1]$, then $A=\left(\mu_{A}, \nu_{A}\right) \in \operatorname{IFCN}(R \times R)$. 
Proposition 3.1. Let $T$ and $C$ be idempotent and $A=\left(\mu_{A}, \nu_{A}\right) \in I F C N(R \times R)$. Then for all $x, y, z \in R$, the following assertions hold.

(1) $A(x, y) \supseteq\left(T\left(\mu_{A}(x z, y z), \mu_{A}(z x, z y)\right), C\left(\nu_{A}(x z, y z), \nu_{A}(z x, z y)\right)\right)$.

(2) $A\left(x^{-1}, y^{-1}\right)=A(x, y)$.

(3) $A(x, y) \supseteq\left(T\left(\mu_{A}(x+z, y+z), T\left(\mu_{A}(x z, y z), \mu_{A}(z x, z y)\right)\right)\right.$, $\left.C\left(\nu_{A}(x+z, y+z), C\left(\nu_{A}(x z, y z), \nu_{A}(z x, z y)\right)\right)\right)$.

(4) $A(-x,-y)=A(x, y)$.

Proof. Let $x, y, z \in R$. Then

$$
\begin{aligned}
\mu_{A}(x, y) & =\mu_{A}\left(x z z^{-1}, y z z^{-1}\right) \geq T\left(\mu_{A}(x z, y z), \mu_{A}\left(z^{-1}, z^{-1}\right)\right) \\
& =T\left(\mu_{A}(x z, y z), 1\right)=\mu_{A}(x z, y z)
\end{aligned}
$$

and

$$
\begin{aligned}
\mu_{A}(x, y) & =\mu_{A}\left(z^{-1} z x, z^{-1} z y\right) \geq T\left(\mu_{A}\left(z^{-1}, z^{-1}\right), \mu_{A}(z x, z y)\right) \\
& =T\left(1, \mu_{A}(z x, z y)\right)=\mu_{A}(z x, z y) .
\end{aligned}
$$

Then from $(a)$ and $(b)$ we get

$$
\mu_{A}(x, y)=T\left(\mu_{A}(x, y), \mu_{A}(x, y)\right) \geq T\left(\mu_{A}(x z, y z), \mu_{A}(z x, z y)\right) .
$$

Also,

$$
\begin{aligned}
\nu_{A}(x, y) & =\nu_{A}\left(x z z^{-1}, y z z^{-1}\right) \leq C\left(\nu_{A}(x z, y z), \nu_{A}\left(z^{-1}, z^{-1}\right)\right) \\
& =C\left(\nu_{A}(x z, y z), 0\right)=\nu_{A}(x z, y z)
\end{aligned}
$$

and

$$
\begin{aligned}
\nu_{A}(x, y) & =\nu_{A}\left(z^{-1} z x, z^{-1} z y\right) \leq C\left(\nu_{A}\left(z^{-1}, z^{-1}\right), \nu_{A}(z x, z y)\right) \\
& =C\left(0, \nu_{A}(z x, z y)\right)=\nu_{A}(z x, z y)
\end{aligned}
$$

Then from $(d)$ and $(e)$ we get

$$
\nu_{A}(x, y)=C\left(\nu_{A}(x, y), \nu_{A}(x, y)\right) \leq C\left(\nu_{A}(x z, y z), \nu_{A}(z x, z y)\right) .
$$

Now from $(c)$ and $(f)$ we get

$$
\begin{gathered}
A(x, y)=\left(\mu_{A}(x, y), \nu_{A}(x, y)\right) \\
\supseteq\left(T\left(\mu_{A}(x z, y z), \mu_{A}(z x, z y)\right), C\left(\nu_{A}(x z, y z), \nu_{A}(z x, z y)\right)\right) .
\end{gathered}
$$

(2) From (a) and (b) in (1) we have

$$
\begin{aligned}
\mu_{A}\left(x^{-1}, y^{-1}\right) & \geq \mu_{A}\left(x x^{-1}, x y^{-1}\right)=\mu_{A}\left(1_{R}, x y^{-1}\right) \geq \mu_{A}\left(1_{R} y, x y^{-1} y\right) \\
& =\mu_{A}(y, x)=\mu_{A}(x, y) \geq \mu_{A}\left(x^{-1} x, x^{-1} y\right)=\mu\left(1_{R}, x^{-1} y\right) \geq \mu\left(1_{R} y^{-1}, x^{-1} y y^{-1}\right) \\
& =\mu_{A}\left(y^{-1}, x^{-1} 1_{R}\right)=\mu_{A}\left(y^{-1}, x^{-1}\right)=\mu_{A}\left(x^{-1}, y^{-1}\right) .
\end{aligned}
$$


Thus

$$
\mu_{A}\left(x^{-1}, y^{-1}\right)=\mu_{A}(x, y)
$$

Also, from $(d)$ and $(e)$ in (1) we obtain

$$
\begin{aligned}
\nu_{A}\left(x^{-1}, y^{-1}\right) & \leq \nu_{A}\left(x x^{-1}, x y^{-1}\right)=\nu_{A}\left(1_{R}, x y^{-1}\right) \leq \nu_{A}\left(1_{R} y, x y^{-1} y\right) \\
& =\nu_{A}(y, x)=\nu_{A}(x, y) \leq \nu_{A}\left(x^{-1} x, x^{-1} y\right)=\nu_{A}\left(1_{R}, x^{-1} y\right) \leq \nu_{A}\left(1_{R} y^{-1}, x^{-1} y y^{-1}\right) \\
& =\nu_{A}\left(y^{-1}, x^{-1} 1_{R}\right)=\nu_{A}\left(y^{-1}, x^{-1}\right)=\nu_{A}\left(x^{-1}, y^{-1}\right)
\end{aligned}
$$

and then

$$
\nu_{A}\left(x^{-1}, y^{-1}\right)=\nu_{A}(x, y) .
$$

Now, from $(g)$ and $(h)$ we have that

$$
A\left(x^{-1}, y^{-1}\right)=\left(\mu_{A}\left(x^{-1}, y^{-1}\right), \nu_{A}\left(x^{-1}, y^{-1}\right)\right)=\left(\mu_{A}(x, y), \nu_{A}(x, y)\right)=A(x, y) .
$$

(3)

$$
\begin{aligned}
\mu_{A}(x, y) & =\mu_{A}(x+z-z, y+z-z) \geq T\left(\mu_{A}(x+z, y+z), \mu_{A}(-z,-z)\right) \\
& =T\left(\mu_{A}(x+z, y+z), 1\right)=\mu_{A}(x+z, y+z) .
\end{aligned}
$$

Now, by $(a)$ from (1) and $(j)$ we obtain

$$
\mu_{A}(x, y)=T\left(\mu_{A}(x, y), \mu(x, y)\right) \geq T\left(\mu_{A}(x+z, y+z), T\left(\mu_{A}(x z, y z), \mu_{A}(z x, z y)\right)\right) .
$$

Also,

$$
\begin{aligned}
\nu_{A}(x, y) & =\nu_{A}(x+z-z, y+z-z) \leq C\left(\nu_{A}(x+z, y+z), \nu_{A}(-z,-z)\right) \\
& =C\left(\nu_{A}(x+z, y+z), 1\right)=\nu_{A}(x+z, y+z) .
\end{aligned}
$$

Now, by $(f)$ from $(1)$ and $(\ell)$ we obtain

$$
\nu_{A}(x, y)=C\left(\nu_{A}(x, y), \nu_{A}(x, y)\right) \leq C\left(\nu_{A}(x+z, y+z), C\left(\nu_{A}(x z, y z), \nu_{A}(z x, z y)\right)\right) .
$$

Then from $(k)$ and $(m)$ we get

$$
\begin{aligned}
A(x, y)= & \left(\mu_{A}(x, y), \nu_{A}(x, y)\right) \\
\supseteq & \left(T\left(\mu_{A}(x+z, y+z), T\left(\mu_{A}(x z, y z), \mu_{A}(z x, z y)\right)\right),\right. \\
& \left.C\left(\nu_{A}(x+z, y+z), C\left(\nu_{A}(x z, y z), \nu_{A}(z x, z y)\right)\right)\right) .
\end{aligned}
$$

(4) By ( $j$ ) from (3) we have

$$
\begin{aligned}
\mu_{A}(x, y) & \geq \mu_{A}(x-y, y-y)=\mu_{A}\left(x-y, 0_{R}\right) \geq \mu_{A}\left(-x+x-y, 0_{R}-x\right) \\
& =\mu_{A}(-y,-x)=\mu_{A}(-x,-y) \geq \mu_{A}(x-x, x-y)=\mu_{A}\left(0_{R}, x-y\right) \\
& \geq \mu_{A}\left(y+0_{R}, y+x-y\right)=\mu_{A}(y, x)=\mu_{A}(x, y)
\end{aligned}
$$


and then

$$
\mu_{A}(x, y)=\mu_{A}(-x,-y)
$$

Also, by $(\ell)$ from (3) we get

$$
\begin{aligned}
\nu_{A}(x, y) & \leq \nu_{A}(x-y, y-y)=\nu_{A}\left(x-y, 0_{R}\right) \leq \nu_{A}\left(-x+x-y, 0_{R}-x\right) \\
& =\nu_{A}(-y,-x)=\nu_{A}(-x,-y) \leq \nu_{A}(x-x, x-y)=\nu_{A}\left(0_{R}, x-y\right) \\
& \leq \nu_{A}\left(y+0_{R}, y+x-y\right)=\nu_{A}(y, x)=\nu_{A}(x, y) .
\end{aligned}
$$

Therefore,

$$
\nu_{A}(x, y)=\nu_{A}(-x,-y)
$$

Thus, from $(n)$ and $(o)$ we get that

$$
A(-x,-y)=\left(\mu_{A}(-x,-y), \nu_{A}(-x,-y)\right)=\left(\mu_{A}(x, y), \nu_{A}(x, y)\right)=A(x, y) .
$$

Definition 3.4. Let $A=\left(\mu_{A}, \nu_{A}\right) \in \operatorname{IFCN}(R \times R)$ and $a \in R$. Define a fuzzy subset $A_{a}=\left(\mu_{A_{a}}, \nu_{A_{a}}\right) \in \operatorname{IFS}(R)$ as $\mu_{A_{a}}(x)=\mu_{A}(a, x)$ and $\nu_{A_{a}}(x)=\nu_{A}(a, x)$ for all $x \in R$. We denote the set of fuzzy subsets $A_{a}=\left(\mu_{A_{a}}, \nu_{A_{a}}\right) \in I F S(R)$ by

$$
\frac{R}{A}=\left(\frac{R}{\mu_{A}}, \frac{R}{\nu_{A}}\right)=\left\{A_{a}=\left(\mu_{A_{a}}, \nu_{A_{a}}\right): a \in R\right\} .
$$

Proposition 3.2. Let $A=\left(\mu_{A}, \nu_{A}\right) \in \operatorname{IFCN}(R \times R)$ and $a \in R$. Then $A_{a}(x)=A_{0_{R}}(x-a)$ for all $x \in R$.

Proof. We prove that $A_{a}(x)=\left(\mu_{A_{a}}(x), \nu_{A_{a}}(x)\right)=A_{0_{R}}(x-a)=\left(\mu_{A_{0_{R}}}(x), \nu_{A_{0_{R}}}(x)\right)$. Now

$$
\begin{aligned}
\mu_{A_{0_{R}}}(x-a) & =\mu_{A}\left(0_{R}, x-a\right)=\mu_{A}(a-a, x-a) \geq T\left(\mu_{A}(a, x), \mu_{A}(-a,-a)\right) \\
& =T\left(\mu_{A}(a, x), \mu_{A}(a, a)\right)=T\left(\mu_{A}(a, x), 1\right)=\mu_{A}(a, x)=\mu_{A}\left(a+0_{R}, x-a+a\right) \\
& \geq T\left(\mu_{A}\left(0_{R}, x-a\right), \mu_{A}(a, a)\right)=T\left(\mu_{A}\left(0_{R}, x-a\right), 1\right)=\mu_{A}\left(0_{R}, x-a\right)=\mu_{0_{R}}(x-a) .
\end{aligned}
$$

Thus,

$$
\mu_{A_{0_{R}}}(x-a)=\mu_{A}(a, x)=\mu_{A_{a}}(x) .
$$

Also,

$$
\begin{aligned}
\nu_{A_{0_{R}}}(x-a) & =\nu_{A}\left(0_{R}, x-a\right)=\nu_{A}(a-a, x-a) \leq C\left(\nu_{A}(a, x), \nu_{A}(-a,-a)\right) \\
& =C\left(\nu_{A}(a, x), \nu_{A}(a, a)\right)=C\left(\nu_{A}(a, x), 0\right)=\nu_{A}(a, x)=\nu_{A}\left(a+0_{R}, x-a+a\right) \\
& \leq C\left(\nu_{A}\left(0_{R}, x-a\right), \nu_{A}(a, a)\right)=C\left(\nu_{A}\left(0_{R}, x-a\right), 0\right)=\nu_{A}\left(0_{R}, x-a\right)=\nu_{A_{0_{R}}}(x-a) .
\end{aligned}
$$

Then

$$
\nu_{A_{0_{R}}}(x-a)=\nu_{A}(a, x)=\nu_{A_{a}}(x) .
$$

Therefore,

$$
A_{a}(x)=\left(\mu_{A_{a}}(x), \nu_{A_{a}}(x)\right)=A_{0_{R}}(x-a)=\left(\mu_{A_{0_{R}}}(x), \nu_{A_{0_{R}}}(x)\right) .
$$


Definition 3.5. Let $A=\left(\mu_{A}, \nu_{A}\right) \in \operatorname{IFS}(R)$, then $A=\left(\mu_{A}, \nu_{A}\right)$ is an intuitionistic fuzzy ideal of $R$ under norms ( $T$ and $C$ ), if the following conditions are satisfied for all $x, y \in R$ :

(1) $\mu_{A}(x+y) \geq T\left(\mu_{A}(x), \mu_{A}(y)\right)$;

(2) $\mu_{A}(x y) \geq T\left(\mu_{A}(x), \mu_{A}(y)\right)$

(3) $\mu_{A}(-x)=\mu_{A}(x)$;

(4) $\mu_{A}\left(0_{R}\right)=1$;

(5) $\mu_{A}(x y) \geq \mu_{A}(x)$ and $\mu_{A}(x y) \geq \mu_{A}(y)$.

(6) $\nu_{A}(x+y) \leq C\left(\nu_{A}(x), \nu_{A}(y)\right)$;

(7) $\nu_{A}(x y) \leq C\left(\nu_{A}(x), \nu_{A}(y)\right)$;

(8) $\nu_{A}(-x)=\nu_{A}(x)$;

(9) $\nu_{A}\left(0_{R}\right)=0$;

(10) $\nu_{A}(x y) \leq \nu_{A}(x)$ and $\nu_{A}(x y) \leq \nu_{A}(y)$.

We denote the set of all intuitionistic fuzzy ideals of $R$ under norms ( $T$ and $C$ ) by $\operatorname{IFIN}(R)$.

Example 3.6. Let $R=\langle\mathbb{R},+,$.$\rangle be a ring of real numbers. Define A=\left(\mu_{A}, \nu_{A}\right) \in I F S(R)$ by

$$
\mu_{A}(x)=\left\{\begin{aligned}
1, & \text { if } x=0_{R} \\
0.65, & \text { otherwise }
\end{aligned}\right.
$$

and

$$
\nu_{A}(x)=\left\{\begin{aligned}
0, & \text { if } x=0_{R} . \\
0.25, & \text { otherwise. }
\end{aligned}\right.
$$

If $T(x, y)=T_{p}(x, y)=x y$ and $C_{b}(x, y)=\min \{1, x+y\}$ for all $x, y \in[0,1]$, Then $A=\left(\mu_{A}, \nu_{A}\right) \in \operatorname{IFIN}(R)$.

Proposition 3.3. Let $A=\left(\mu_{A}, \nu_{A}\right) \in \operatorname{IFCN}(R \times R)$. If $T$ and $C$ are idempotent, then $A_{0_{R}}=\left(\mu_{A_{0_{R}}}, \nu_{A_{0_{R}}}\right) \in \operatorname{IFIN}(R)$.

Proof. Let $A=\left(\mu_{A}, \nu_{A}\right) \in \operatorname{IFCN}(R \times R)$ and $x, y \in R$. Then

$$
\begin{aligned}
\mu_{A_{0_{R}}}(x+y) & =\mu_{A}\left(0_{R}, x+y\right)=\mu_{A}\left(0_{R}+0_{R}, x+y\right) \\
& \geq T\left(\mu_{A}\left(0_{R}, x\right), \mu_{A}\left(0_{R}, y\right)\right)=T\left(\mu_{A_{0_{R}}}(x), \mu_{A_{0_{R}}}(y)\right) .
\end{aligned}
$$

(3) $\mu_{A_{0_{R}}}(-x)=\mu_{A}\left(0_{R},-x\right)=\mu_{A}\left(-0_{R},-x\right)=\mu_{A}\left(0_{R}, x\right)=\mu_{A_{0_{R}}}(x)$

(by Proposition 3.4 (part 4)).

(4) $\mu_{A_{0_{R}}}\left(0_{R}\right)=\mu_{A}\left(0_{R}, 0_{R}\right)=1$.

(5) $\quad \mu_{A_{0_{R}}}(x y)=\mu_{A}\left(0_{R}, x y\right)=\mu_{A}\left(0_{R} y, x y\right) \geq T\left(\mu_{A}\left(0_{R}, x\right), \mu_{A}(y, y)\right)=T\left(\mu_{A}\left(0_{R}, x\right), 1\right)$

$$
=\mu_{A}\left(0_{R}, x\right)=\mu_{A_{0_{R}}}(y) \text {. }
$$

Similarly $\mu_{A_{0_{R}}}(x y) \geq \mu_{A_{0_{R}}}(y)$.

$$
\begin{aligned}
\nu_{A_{0_{R}}}(x+y) & =\nu_{A}\left(0_{R}, x+y\right)=\nu_{A}\left(0_{R}+0_{R}, x+y\right) \\
& \leq C\left(\nu_{A}\left(0_{R}, x\right), \nu_{A}\left(0_{R}, y\right)\right)=C\left(\nu_{A_{0_{R}}}(x), \nu_{A_{0_{R}}}(y)\right)
\end{aligned}
$$




$$
\begin{aligned}
\nu_{A_{0_{R}}}(x y) & =\nu_{A}\left(0_{R}, x y\right)=\nu_{A}\left(0_{R} 0_{R}, x y\right) \\
& \leq C\left(\nu_{A}\left(0_{R}, x\right), \nu_{A}\left(0_{R}, y\right)\right)=C\left(\nu_{A_{0_{R}}}(x), \nu_{A_{0_{R}}}(y)\right) .
\end{aligned}
$$

(8) $\nu_{A_{0_{R}}}(-x)=\nu_{A}\left(0_{R},-x\right)=\nu_{A}\left(-0_{R},-x\right)=\nu_{A}\left(0_{R}, x\right)=\nu_{A_{0_{R}}}(x)$

(by Proposition 3.4 (part 4)).

(9) $\nu_{A_{0_{R}}}\left(0_{R}\right)=\nu_{A}\left(0_{R}, 0_{R}\right)=1$.

$$
\begin{aligned}
\nu_{A_{0_{R}}}(x y) & =\nu_{A}\left(0_{R}, x y\right)=\nu_{A}\left(0_{R} y, x y\right) \leq C\left(\nu_{A}\left(0_{R}, x\right), \nu_{A}(y, y)\right)=C\left(\nu_{A}\left(0_{R}, x\right), 1\right) \\
& =\nu_{A}\left(0_{R}, x\right)=\nu_{A_{0_{R}}}(y) .
\end{aligned}
$$

Similarly, $\nu_{A_{0_{R}}}(x y) \leq \nu_{A_{0_{R}}}(y)$.

Thus, from (1) to (10) we get that $A_{0_{R}}=\left(\mu_{A_{0_{R}}}, \nu_{A_{0_{R}}}\right) \in \operatorname{IFIN}(R)$.

Definition 3.7. Let $A=\left(\mu_{A}, \nu_{A}\right) \in \operatorname{IFS}(R)$. Define $C(A)=\left(C\left(\mu_{A}\right), C\left(\nu_{A}\right)\right) \in \operatorname{IF} S(R \times R)$ as $C\left(\mu_{A}\right)(x, y)=\mu_{A}(x-y)$ and $C\left(\nu_{A}\right)(x, y)=\nu_{A}(x-y)$ for all $x, y \in R$. We call $C(A)$ an intuitionistic fuzzy relation induced by $A$.

Proposition 3.4. If $A=\left(\mu_{A}, \nu_{A}\right) \in \operatorname{IFIN}(R)$, then $C(A) \in \operatorname{IFCN}(R \times R)$.

Proof. Let $A=\left(\mu_{A}, \nu_{A}\right) \in I F I N(R)$ and $x, y, z, t \in R$. Then

(1) $C\left(\mu_{A}\right)(x, x)=\mu_{A}(x-x)=\mu_{A}\left(0_{R}\right)=1$.

(2) $C\left(\mu_{A}\right)(x, y)=\mu_{A}(x-y)=\mu_{A}(-(x-y))=\mu_{A}(y-x)=C\left(\mu_{A}\right)(y, x)$.

$$
\begin{aligned}
C\left(\mu_{A}\right)(x, y) & =\mu_{A}(x-y)=\mu_{A}(x-z+z-y) \\
& \geq T\left(\mu_{A}(x-z), \mu_{A}(z-y)\right)=T\left(C\left(\mu_{A}\right)(x, z), C\left(\mu_{A}\right)(z, y)\right) .
\end{aligned}
$$

$C\left(\mu_{A}\right)(x+z, y+t)=\mu_{A}(x+z-(y+t))=\mu_{A}(x-y+z-t)$

$$
\geq T\left(\mu_{A}(x-y), \mu_{A}(z-t)\right)=T\left(C\left(\mu_{A}\right)(x, y), C\left(\mu_{A}\right)(z, t)\right) .
$$

$$
\begin{aligned}
C\left(\mu_{A}\right)(x z, y t) & =\mu_{A}(x z-(y t))=\mu_{A}((x-y) z+y(z-t)) \\
& \geq T\left(\mu_{A}((x-y) z), \mu_{A}(y(z-t))\right) \geq T\left(\mu_{A}(x-y), \mu_{A}(z-t)\right) \\
& =T\left(C\left(\mu_{A}\right)(x, y), C\left(\mu_{A}\right)(z, t)\right) .
\end{aligned}
$$

(6) $C\left(\nu_{A}\right)(x, x)=\nu_{A}(x-x)=\nu_{A}\left(0_{R}\right)=1$.

(7) $C\left(\nu_{A}\right)(x, y)=\nu_{A}(x-y)=\nu_{A}(-(x-y))=\nu_{A}(y-x)=C\left(\nu_{A}\right)(y, x)$.

$$
\begin{aligned}
C\left(\nu_{A}\right)(x, y) & =\nu_{A}(x-y)=\nu_{A}(x-z+z-y) \\
& \leq C\left(\nu_{A}(x-z), \nu_{A}(z-y)\right)=C\left(C\left(\nu_{A}\right)(x, z), C\left(\nu_{A}\right)(z, y)\right) . \\
C\left(\nu_{A}\right)(x+z, y+t) & =\nu_{A}(x+z-(y+t))=\nu_{A}(x-y+z-t) \\
& \leq C\left(\nu_{A}(x-y), \nu_{A}(z-t)\right)=C\left(C\left(\nu_{A}\right)(x, y), C\left(\nu_{A}\right)(z, t)\right) . \\
C\left(\nu_{A}\right)(x z, y t) & =\nu_{A}(x z-(y t))=\nu_{A}((x-y) z+y(z-t)) \\
& \leq C\left(\nu_{A}((x-y) z), \nu_{A}(y(z-t))\right) \leq C\left(\nu_{A}(x-y), \nu_{A}(z-t)\right) \\
& =C\left(C\left(\nu_{A}\right)(x, y), C\left(\nu_{A}\right)(z, t)\right) .
\end{aligned}
$$

Thus, $C(A) \in I F C N(R \times R)$. 
Proposition 3.5. Let $A=\left(\mu_{A}, \nu_{A}\right) \in \operatorname{IFCN}(R \times R)$ and $a, b \in R$. Then $A_{a}=A_{b}$ if and only if $A_{0_{R}}(a-b)=(1,0)$.

Proof. Let $A_{a}=\left(\mu_{A_{a}}, \nu_{A_{a}}\right)=A_{b}=\left(\mu_{A_{b}}, \nu_{A_{b}}\right)$ and $a, b, x \in R$. Then $\mu_{A_{a}}(x)=\mu_{A_{b}}(x)$ and so $\mu_{A}(a, x)=\mu_{A}(b, x)$. Also $\nu_{A_{a}}(x)=\nu_{A_{b}}(x)$ and so $\nu_{A}(a, x)=\nu_{A}(b, x)$. Now, by Proposition 3.6 we get that $\mu_{A_{0_{R}}}(a-b)=\mu_{A}(b, a)=\mu_{A}(a, a)=1$ and $\nu_{A_{0_{R}}}(a-b)=\nu_{A}(b, a)=\nu_{A}(a, a)=0$. Thus

$$
A_{0_{R}}(a-b)=\left(\mu_{A_{0_{R}}}(a-b), \nu_{A_{0_{R}}}(a-b)\right)=(1,0) .
$$

Conversely, let $A_{0_{R}}(a-b)=\left(\mu_{A_{0_{R}}}(a-b), \nu_{A_{0_{R}}}(a-b)\right)=(1,0)$. Firstly, we show that $\mu_{A_{a}}=\mu_{A_{b}}$. Since

$$
\begin{aligned}
\mu_{A_{a}}(x) & =\mu_{A}(a, x)=\mu_{A}\left(0_{R}+a, x-a+a\right) \\
& \geq T\left(\mu_{A}\left(0_{R}, x-a\right), \mu(a, a)\right)=T\left(\mu_{A}\left(0_{R}, x-a\right), 1\right)=\mu_{A}\left(0_{R}, x-a\right) \\
& \geq T\left(\mu_{A}\left(0_{R}, b-a\right), \mu_{A}(b-a, x-a)\right)=T\left(1, \mu_{A}(b-a, x-a)\right) \\
& =\mu_{A}(b-a, x-a)=\mu_{A}(x-a, b-a) \\
& \geq T\left(\mu_{A}(x, b), \mu_{A}(-a,-a)\right)=T\left(\mu_{A}(x, b), 1\right)=\mu_{A}(x, b) \\
& =\mu_{A_{b}}(x)
\end{aligned}
$$

and then $\mu_{A_{a}} \supseteq \mu_{A_{b}}$. Also, by symmetry, we obtain that $\mu_{A_{a}} \subseteq \mu_{A_{b}}$. Therefore, $\mu_{A_{a}}=\mu_{A_{b}}$.

Also

$$
\begin{aligned}
\nu_{A_{a}}(x) & =\nu_{A}(a, x)=\nu_{A}\left(0_{R}+a, x-a+a\right) \\
& \leq C\left(\nu_{A}\left(0_{R}, x-a\right), \nu_{A}(a, a)\right)=C\left(\nu_{A}\left(0_{R}, x-a\right), 0\right)=\nu_{A}\left(0_{R}, x-a\right) \\
& \leq C\left(\nu_{A}\left(0_{R}, b-a\right), \nu_{A}(b-a, x-a)\right)=C\left(0, \nu_{A}(b-a, x-a)\right) \\
& =\nu_{A}(b-a, x-a)=\nu_{A}(x-a, b-a) \\
& \leq C\left(\nu_{A}(x, b), \nu_{A}(-a,-a)\right)=C\left(\nu_{A}(x, b), 0\right)=\nu_{A}(x, b) \\
& =\nu_{A_{b}}(x)
\end{aligned}
$$

and so $\nu_{A_{a}} \subseteq \nu_{A_{b}}$. Also by symmetry, we obtain that $\nu_{A_{a}} \supseteq \nu_{A_{b}}$. Therefore, $\nu_{A_{a}}=\nu_{A_{b}}$.

Thus $A_{a}=\left(\mu_{A_{a}}, \nu_{A_{a}}\right)=A_{b}=\left(\mu_{A_{b}}, \nu_{A_{b}}\right)$.

Corollary 3.8. Let $A=\left(\mu_{A}, \nu_{A}\right) \in \operatorname{IFCN}(R \times R)$ and $a, b \in R$. Then $A_{a}=A_{b}$ if and only if $A(a, b)=(1,0)$.

Proof. From Proposition 3.12 we have $A_{a}=A_{b}$ if and only if $A_{0_{R}}(a-b)=(1,0)$, if and only if $\mu_{A_{0_{R}}}(a-b)=1$ and $\nu_{A_{0_{R}}}(a-b)=0$, if and only if $\mu_{A_{a}}(b)=1$ and $\nu_{A_{a}}(b)=0$, if and only if $\mu_{A}(a, b)=1$ and $\nu_{A}(a, b)=0$, if and only if $A(a, b)=\left(\mu_{A}(a, b), \nu_{A}(a, b)\right)=(1,0)$.

Definition 3.9. Let $A=\left(\mu_{A}, \nu_{A}\right) \in \operatorname{IFCN}(R \times R)$ and $a, b \in R$. Define addition

$$
A_{a} \oplus A_{b}=\left(\mu_{A_{a}} \oplus \mu_{A_{b}}, \nu_{A_{a}} \oplus \nu_{A_{b}}\right): R \rightarrow[0,1]
$$

by 


$$
\left(\mu_{A_{a}} \oplus \mu_{A_{b}}\right)(x)=\left\{\begin{aligned}
\sup _{x=y+z} T\left(\mu_{A_{a}}(y), \mu_{A_{b}}(z)\right), & \text { if } x=y+z \\
0, & \text { otherwise }
\end{aligned}\right.
$$

and

$$
\left(\nu_{A_{a}} \oplus \nu_{A_{b}}\right)(x)=\left\{\begin{aligned}
\inf _{x=y+z} C\left(\nu_{A_{a}}(y), \nu_{A_{b}}(z)\right), & \text { if } x=y+z . \\
0, & \text { otherwise. }
\end{aligned}\right.
$$

Also, define product

$$
A_{a} \odot A_{b}=\left(\mu_{A_{a}} \odot \mu_{A_{b}}, \nu_{A_{a}} \odot \nu_{A_{b}}\right): R \rightarrow[0,1]
$$

by

$$
\left(\mu_{A_{a}} \odot \mu_{A_{b}}\right)(x)=\left\{\begin{array}{rr}
\sup _{x=y z} T\left(\mu_{A_{a}}(y), \mu_{A_{b}}(z)\right), & \text { if } x=y z . \\
0, & \text { otherwise. }
\end{array}\right.
$$

and

$$
\left(\nu_{A_{a}} \odot \nu_{A_{b}}\right)(x)=\left\{\begin{aligned}
\inf _{x=y z} C\left(\mu_{A_{a}}(y), \mu_{A_{b}}(z)\right) & \text { if } x=y z \\
0 & \text { otherwise. }
\end{aligned}\right.
$$

Proposition 3.6. Let $A=\left(\mu_{A}, \nu_{A}\right) \in \operatorname{IFCN}(R \times R)$ and $a, b \in R$. Then $A_{a} \oplus A_{b}=A_{a+b}$.

Proof. Firstly, we prove the binary operation $\oplus$ is well-defined. Let $A_{a} \oplus A_{b}=A_{c} \oplus A_{d}$, then $A_{a}=A_{c}$ and $A_{b}=A_{d}$. Now, by Corollary 3.13 we get that $A(a, c)=(1,0)$ and $A(b, d)=(1,0)$. Then $\mu_{A}(a, c)=1$ and $\nu_{A}(a, c)=0$ and $\mu_{A}(b, d)=1$ and $\nu_{A}(b, d)=0$. Then

$$
\mu_{A}(a+b, c+d) \geq T\left(\mu_{A}(a, c), \mu_{A}(b, d)\right)=T(1,1)=1
$$

and so $\mu_{A}(a+b, c+d)=1$. Also,

$$
\nu_{A}(a+b, c+d) \leq C\left(\nu_{A}(a, c), \nu_{A}(b, d)\right)=C(0,0)=0
$$

and then $\nu_{A}(a+b, c+d)=0$. Thus

$$
A(a+b, c+d)=\left(\mu_{A}(a+b, c+d), \nu_{A}(a+b, c+d)\right)=(1,0)
$$

and by Corollary 3.13 we obtain that $A_{a+b}=A_{c+d}$ and so the binary operation $\oplus$ is well-defined. Now, we prove $A_{a} \oplus B_{b}=A_{a+b}$. Let $x \in R$ be such that $x=y+z$. Then

$$
\begin{aligned}
\left(\mu_{A_{a}} \oplus \mu_{A_{b}}\right)(x) & =\sup _{x=y+z} T\left(\mu_{A_{a}}(y), \mu_{A_{b}}(z)\right) \\
& \geq \sup _{x=y+z, \mu_{A_{a}}(y) \neq \mu_{A_{b}}(z)} T\left(\mu_{A_{a}}(y), \mu_{A_{b}}(z)\right) \\
& =\sup _{x=y+z, \mu_{A_{a}}(y) \neq \mu_{A_{b}}(z)} T\left(\mu_{A_{0_{R}}}(y-a), \mu_{A_{0_{R}}}(z-b)\right) \\
& =\mu_{A_{0_{R}}}((y+z)-(a+b))=\mu_{A_{0_{R}}}((x)-(a+b))=\mu_{A_{a}}(a+b, x)=\mu_{A_{a+b}}(x) .
\end{aligned}
$$


Then $\mu_{A_{a}} \oplus \mu_{A_{b}} \supseteq \mu_{A_{a+b}}$. Conversely, for each $y, z \in R$ satisfies $x=y+z$ we get

$$
\begin{aligned}
\mu_{A_{a+b}}(x) & =\mu_{A_{0_{R}}}(x-(a+b)) \\
& =\mu_{A_{0_{R}}}(y+z-(a+b))=\mu_{A_{0_{R}}}((y-a)+(z-b)) \\
& \geq T\left(A_{0_{R}}(y-a), \mu_{A_{0_{R}}}(z-b)\right) \\
& =T\left(\mu_{A_{a}}(y), \mu_{A_{b}}(z)\right) .
\end{aligned}
$$

Thus $\mu_{A_{a+b}}(x) \geq \sup _{x=y+z} T\left(\mu_{A_{a}}(y), \mu_{A_{b}}(z)\right)$ and so $\mu_{A_{a+b}} \supseteq \mu_{A_{a}} \oplus \mu_{A_{b}}$. Then

$$
\mu_{A_{a}} \oplus \mu_{A_{b}}=\mu_{A_{a+b}}
$$

Also,

$$
\begin{aligned}
\left(\nu_{A_{a}} \oplus \nu_{A_{b}}\right)(x) & =\inf _{x=y+z} C\left(\nu_{A_{a}}(y), \nu_{A_{b}}(z)\right) \\
& \leq \inf _{x=y+z, \nu_{A_{a}}(y) \neq \nu_{A_{b}}(z)} C\left(\nu_{A_{a}}(y), \nu_{A_{b}}(z)\right) \\
& =\inf _{x=y+z, \nu_{A_{a}}(y) \neq \nu_{A_{b}}(z)} C\left(\nu_{A_{0_{R}}}(y-a), \nu_{A_{0_{R}}}(z-b)\right) \\
& =\nu_{A_{0_{R}}}((y+z)-(a+b))=\nu_{A_{0_{R}}}((x)-(a+b))=\nu_{A_{a}}(a+b, x)=\nu_{A_{a+b}}(x) .
\end{aligned}
$$

Then $\nu_{A_{a}} \oplus \nu_{A_{b}} \subseteq \nu_{A_{a+b}}$. Conversely, for each $y, z \in R$ which satisfy $x=y+z$ we get

$$
\begin{aligned}
\nu_{A_{a+b}}(x) & =\nu_{A_{0_{R}}}(x-(a+b)) \\
& =\nu_{A_{0_{R}}}(y+z-(a+b))=\nu_{A_{0_{R}}}((y-a)+(z-b)) \\
& \leq C\left(A_{A_{R}}(y-a), \nu_{A_{0_{R}}}(z-b)\right) \\
& =C\left(\nu_{A_{a}}(y), \nu_{A_{b}}(z)\right) .
\end{aligned}
$$

Thus, $\nu_{A_{a+b}}(x) \leq \inf _{x=y+z} C\left(\nu_{A_{a}}(y), \nu_{A_{b}}(z)\right)$ and so $\nu_{A_{a+b}} \subseteq \nu_{A_{a}} \oplus \nu_{A_{b}}$. Then

$$
\nu_{A_{a}} \oplus \nu_{A_{b}}=\nu_{A_{a+b}} .
$$

Thus, from $(p)$ and $(q)$ we get that

$$
A_{a} \oplus A_{b}=\left(\mu_{A_{a}} \oplus \mu_{A_{b}}, \nu_{A_{a}} \oplus \nu_{A_{b}}\right)=\left(\mu_{A_{a+b}}, \nu_{A_{a+b}}\right)=A_{a+b}
$$

Proposition 3.7. Let $A=\left(\mu_{A}, \nu_{A}\right) \in \operatorname{IFCN}(R \times R)$ and $a, b \in R$. Then $A_{a} \odot A_{b}=A_{a b}$.

Proof. First, we prove the binary operation $\odot$ is well-defined. Let $A_{a} \odot A_{b}=A_{c} \odot A_{d}$. Then $A_{a}=A_{c}$ and $A_{b}=A_{d}$. Now, by Corollary 3.13 we get that $A(a, c)=(1,0)$ and $A(b, d)=(1,0)$. Then $\mu_{A}(a, c)=1$ and $\nu_{A}(a, c)=0$ and $\mu_{A}(b, d)=1$ and $\nu_{A}(b, d)=0$. Then

$$
\mu_{A}(a b, c d) \geq T\left(\mu_{A}(a, c), \mu_{A}(b, d)\right)=T(1,1)=1
$$

and so $\mu_{A}(a b, c d)=1$. Also,

$$
\nu_{A}(a b, c d) \leq C\left(\nu_{A}(a, c), \nu_{A}(b, d)\right)=C(0,0)=0
$$


and then $\nu_{A}(a b, c d)=0$. Thus,

$$
A(a b, c d)=\left(\mu_{A}(a b, c d), \nu_{A}(a b, c d)\right)=(1,0)
$$

and by Corollary 3.13 we obtain that $A_{a b}=A_{c d}$ and so the binary operation $\odot$ is well-defined. Let $x \in R$ be such that $x=y z$. Then,

$$
\begin{aligned}
\mu_{A_{a b}}(x) & =\mu_{A}(a b, x)=\mu_{A}(a b, y z) \\
& \geq T\left(\mu_{A}(a, y), \mu_{A}(b, z)\right)=T\left(\mu_{A_{a}}(y), \mu_{A_{b}}(z)\right) \\
& =\sup _{x=y z, \mu_{A_{a}}(y) \neq \mu_{A_{b}}(z)} T\left(\mu_{A_{a}}(y), \mu_{A_{b}}(z)\right)=\left(\mu_{A_{a}} \odot \mu_{A_{b}}\right)(x)=\sup _{x=y z} T\left(\mu_{A_{a}}(y), \mu_{A_{b}}(z)\right) \\
& \geq \sup _{x=y z, \mu_{A_{a}}(y) \neq \mu_{A_{b}}(z)} T\left(\mu_{A_{a}}(y), \mu_{A_{b}}(z)\right)=\sup _{x=y z, \mu_{A_{a}}(y) \neq \mu_{A_{b}}(z)} T\left(\mu_{A_{0_{R}}}(y-a), \mu_{A_{0_{R}}}(z-b)\right) \\
& =\mu_{A_{0_{R}}}(y z-a b)=\mu_{A_{0_{R}}}(x-a b)=\mu_{A}(a b, x)=\mu_{A_{a b}}(x) .
\end{aligned}
$$

Thus,

$$
\mu_{A_{a}} \odot \mu_{A_{b}}=\mu_{A_{a b}}
$$

Also,

$$
\begin{aligned}
\nu_{A_{a b}}(x) & =\nu_{A}(a b, x)=\nu_{A}(a b, y z) \\
& \leq C\left(\nu_{A}(a, y), \nu_{A}(b, z)\right)=C\left(\nu_{A_{a}}(y), \nu_{A_{b}}(z)\right) \\
& =\sup _{x=y z, \nu_{A_{a}}(y) \neq \nu_{A_{b}}(z)} C\left(\nu_{A_{a}}(y), \nu_{A_{b}}(z)\right)=\left(\mu_{A_{a}} \odot \mu_{A_{b}}\right)(x)=\sup _{x=y z} C\left(\mu_{A_{a}}(y), \mu_{A_{b}}(z)\right) \\
& \leq \sup _{x=y z, \nu_{A_{a}}(y) \neq \nu_{A_{b}}(z)} C\left(\nu_{A_{a}}(y), \nu_{A_{b}}(z)\right)=\sup _{x=y z, \nu_{A_{a}}(y) \neq \nu_{A_{b}}(z)} C\left(\nu_{A_{0_{R}}}(y-a), \nu_{A_{0_{R}}}(z-b)\right) \\
& =\nu_{A_{0_{R}}}(y z-a b)=\nu_{A_{0_{R}}}(x-a b)=\nu_{A}(a b, x)=\nu_{A_{a b}}(x) .
\end{aligned}
$$

Thus,

$$
\nu_{A_{a}} \odot \nu_{A_{b}}=\nu_{A_{a b}}
$$

Then, from $(r)$ and $(s)$ we get

$$
A_{a} \odot A_{b}=\left(\mu_{A_{a}} \odot \mu_{A_{b}}, \nu_{A_{a}} \odot \nu_{A_{b}}\right)=\left(\mu_{A_{a b}}, \nu_{A_{a b}}\right)=A_{a b} .
$$

Proposition 3.8. If $A=\left(\mu_{A}, \nu_{A}\right) \in \operatorname{IFCN}(R \times R)$, then

$$
\left(\frac{R}{A}=\left\{A_{a}=\left(\mu_{A_{a}}, \nu_{A_{a}}\right): a \in R\right\}, \oplus, \odot\right)
$$

is a ring.

Proof. Let $a, b, c \in R$. As in Propositions 3.15 and 3.16 we get that $A_{a} \oplus A_{b} \in \frac{R}{A}$ and $A_{a} \odot A_{b} \in \frac{R}{A}$. It is easy to prove that $\left(\frac{R}{A}, \oplus\right)$ is an Abelian group. Now

(1) $A_{a} \odot\left(A_{b} \odot A_{c}\right)=A_{a} \odot A_{b c}=A_{a(b c)}=A_{(a b) c}=\left(A_{a} \odot A_{b}\right) \odot A_{c}$ (associative multiplication). 
(2) $A_{a} \odot\left(A_{b} \oplus A_{c}\right)=A_{a} \odot A_{b+c}=A_{a(b+c)}=A_{a b+a c}=A_{a b} \oplus A_{a c}=\left(A_{a} \odot A_{b}\right) \oplus\left(A_{a} \odot A_{c}\right)$ (distributive laws).

(3) $\left(A_{b} \oplus A_{c}\right) \odot A_{a}=A_{b+c} \odot A_{a}=A_{(b+c) a}=A_{b a+c a}=A_{b a} \oplus A_{c a}=\left(A_{b} \odot A_{a}\right) \oplus\left(A_{c} \odot A_{a}\right)$ (distributive laws). Then $\left(\frac{R}{A}, \oplus, \odot\right)$ will be a ring.

If $R$ is a commutative ring, then $A_{a} \odot A_{b}=A_{a b}=A_{b a}=A_{b} \odot A_{a}$ and so $\left(\frac{R}{A}, \oplus, \odot\right)$ is a commutative ring.

If $R$ be a ring with identity $1_{R}$, then $A_{a} \odot A_{1_{R}}=A_{a 1_{R}}=A_{a}$ and thus $\left(\frac{R}{A}, \oplus, \odot\right)$ is a ring with identity $A_{1_{R}}$.

\section{Acknowledgement}

We would like to thank the reviewers for carefully reading the manuscript and making several helpful comments to increase the quality of the paper.

\section{References}

[1] Abu Osman, M. T. (1987). On some products of fuzzy subgroups. Fuzzy Sets and Systems, $24,79-86$.

[2] Atanassov, K. (1986). Intuitionistic fuzzy sets. Fuzzy Sets and Systems, 20, 87-96.

[3] Buckley, J. J., \& Eslami, E. (2002). An Introduction to Fuzzy Logic and Fuzzy Sets, Springer-Verlag Berlin Heidelberg GmbH.

[4] Dubois, D., \& Prade, H. (1988). Fuzzy Sets and Systems, Academic Press, New York.

[5] Klaua, D. (2010). An early approach toward graded identity and graded membership in set theory. Fuzzy Sets and Systems, 161, 2369-2379.

[6] Liang, R., Lu, S., Wang, X., Lu, Y., Mandal, V., Patacsil, D., \& Kumar, D. (2006). A Fuzzy-Set-Theory-Based Approach to Differential Gene Expression Data Analysis. BMC Bioinformatics, 7 (Suppl 4): S7. 2006.

[7] Malik, D. S., Mordeson, J. N., \& Sen, M. K. (1997). Fundamentals of Abstract Algebra, McGraw Hill.

[8] Rasuli, R. (2016). Fuzzy Ideals of Subtraction Semigroups with Respect to A $t$-norm and A t-conorm. The Journal of Fuzzy Mathematics, Los Angeles, 24(4) , 881-892.

[9] Rasuli, R. (2016). Fuzzy modules over a t-norm. Int. J. Open Problems Compt. Math., 9(3), $12-18$.

[10] Rasuli, R. (2016). Fuzzy Subrings over a $t$-norm. The Journal of Fuzzy Mathematics, Los Angeles, 24(4), 995-1000. 
[11] Rasuli, R. (2016). Norms over intuitionistic fuzzy subrings and ideals of a ring. Notes on Intuitionistic Fuzzy Sets, 22(5), 72-83.

[12] Rasuli, R. (2017). Norms over fuzzy Lie algebra. Journal of New Theory, 15, 32-38.

[13] Rasuli, R. (2017). Fuzzy subgroups on direct product of groups over a $t$-norm. Journal of Fuzzy Set Valued Analysis, 3, 96-101.

[14] Rasuli, R. (2017). Characterizations of intuitionistic fuzzy subsemirings of semirings and their homomorphisms by norms. Journal of New Theory, 18, 39-52.

[15] Rasuli, R. (2017). Intuitionistic fuzzy subrings and ideals of a ring under norms, LAP LAMBERT Academic publishing, ISBN: 978-620-2-06926-7.

[16] Rasuli, R. (2018). Characterization of Q-Fuzzy subrings (Anti Q-Fuzzy Subrings) with respect to a $T$-norm (T-Conorms). Journal of Information and Optimization Science, 31, $1-11$.

[17] Rasuli, R. (2018). T-Fuzzy Submodules of $R \times M$. Journal of New Theory, 22, 92-102.

[18] Rasuli, R. (2018). Fuzzy subgroups over a T-norm. Journal of Information and Optimization Science, 39, 1757-1765.

[19] Rasuli, R. (2018). Fuzzy Sub-vector Spaces and Sub-bivector Spaces under $t$-Norms. General Letters in Mathematics, 5, 47-57.

[20] Rasuli, R. (2019). Anti Fuzzy Submodules over a $t$-conorm and Some of Their Properties. The Journal of Fuzzy Mathematics Los Angles, 27, 229-236.

[21] Rasuli, R. (2019). Artinian and Noetherian Fuzzy Rings. International Journal of Open Problems in Computer Science and Mathematics, 12, 1-7.

[22] Rasuli, R., \& Narghi, H. (2019). T-Norms Over Q-Fuzzy Subgroups of Group. Jordan Journal of Mathematics and Statistics (JJMS), 12, 1-13.

[23] Rasuli, R. (2019). Fuzzy equivalence relation, fuzzy congrunce relation and fuzzy normal subgroups on group $G$ over $t$-norms. Asian Journal of Fuzzy and Applied Mathematics, 7 , $14-28$.

[24] Rasuli, R. (2019). Norms over anti fuzzy G-submodules, MathLAB Journal, 2, 56-64.

[25] Rasuli, R. (2019). Norms over bifuzzy bi-ideals with operators in semigroups. Notes on Intuitionistic Fuzzy Sets, 25, 1-11.

[26] Rasuli, R.(2019). Norms Over Basic Operations on Intuitionistic Fuzzy Sets. The Journal of Fuzzy Mathematics, Los Angles, 27(3), 561-582.

[27] Rasuli, R. (2019). T-fuzzy Bi-ideals in Semirings. Earthline Journal of Mathematical Sciences, 27(1), 241-263. 
[28] Rasuli, R. (2019). Norms Over Intuitionistic Fuzzy Vector Spaces. Algebra Letters, 1(1), $1-19$.

[29] Rasuli, R. (2019). Some Results of Anti Fuzzy Subrings Over t-Conorms. MathLAB Journal, 1(4), 25-32.

[30] Rasuli, R. (2020). Anti Fuzzy Equivalence Relation on Rings with respect to $t$-conorm $C$. Earthline Journal of Mathematical Sciences, 3(1), 1-19.

[31] Rasuli, R. (2020). Anti Fuzzy Subbigroups of Bigroups under t-conorms. The Journal of Fuzzy Mathematics, Los Angles, 28(1), 181-200.

[32] Rasuli, R. (2020). $t$-norms over Fuzzy Multigroups. Earthline Journal of Mathematical Sciences, 3(2), 207-228.

[33] Rasuli, R. (2020). Anti Q-fuzzy subgroups under t-conorms. Earthline Journal of Mathematical Sciences, 4(1), 13-28.

[34] Rasuli, R. (2020). Anti Fuzzy Congruence on Product Lattices with respect to $S$-norms. The Second National Congress on Mathematics and Statistics, Conbad Kavous University.

[35] Rasuli, R. (2020). Direct product of fuzzy multigroups under $t$-norms. Open Journal of Discrete Applied Mathematics (ODAM), 3(1), 75-85.

[36] Rasuli, R. (2020). Level subsets and translations of QF ST(G). MathLAB Journal, 5(1), $1-11$.

[37] Rasuli, R. (2020). Conorms over anti fuzzy vector spaces. Open Journal of Mathematical Sciences, 4, 158-167.

[38] Rasuli, R. (2020). Intuitionistic fuzzy subgroups with respect to norms (T, S). Eng. Appl. Sci. Lett. (EASL), 3(2), 40-53.

[39] Rasuli, R., Moatamedi Nezhad, M., \& Naraghi, H. (2020). Characterization of TF(G) and direct product of it. The First National Conference on Soft Computing and Cognitive Science, Conbad Kavous University, July 2020.

[40] Rasuli, R. (2020). Anti complex fuzzy subgroups under s-norms.Eng. Appl. Sci. Lett. (EASL), 3(4), 1-10.

[41] Rasuli, R., \& Moatamedi Nezhad, M. M. (2020). Characterization of Fuzzy modules and anti fuzzy modules under norms. The First International Conference on Basic Sciences, Tehran, October 21.

[42] Rasuli R., \& Moatamedi Nezhad, M. M. (2020). Fuzzy subrings and anti fuzzy subrings under norms. The First International Conference on Basic Sciences, Tehran, October 21. 
[43] Rasuli, R. (2021). Anti $Q$-fuzzy translations of anti $Q$-soft subgroups. The Third national Conference on Managent and Fuzzy Systems, University of Eyvanekey, March 2021.

[44] Rasuli, R. (2021). Conorms over conjugates and generalized characterestics of anti $Q$-fuzzy subgroups. The Third national Conference on Managent and Fuzzy Systems, University of Eyvanekey, March 2021.

[45] Rasuli, R. (2021). Fuzzy congruence on product lattices under T-norms. Journal of Information and Optimization Sciences, 42(2), 333-343.

[46] Rasuli, R. (2021). Intuitionistic fuzzy congruences on product lattices under norms. Journal of Interdisciplinary Mathematics, 24(2), 1-24.

[47] Rasuli, R. (2021). Conorms over level subsets and translations of anti $Q$-fuzzy Subgroups. International Journal of Mathematics and Computation, 32(2), 55-67.

[48] Zadeh, L. A. (1965). Fuzzy sets. Information and Control, 8, 338-353. 